\title{
Immigration européenne de retraités vers la « Riviera turque » : le cas d'Alanya (côte méditerranéenne)
}

\section{Ilkay Südaş et Mustafa Mutluer}

Traducteur : Stéphane de Tapia

\section{(2) OpenEdition}

Journals

Édition électronique

URL : https://journals.openedition.org/remi/3381

DOI : $10.4000 /$ remi.3381

ISSN : $1777-5418$

Éditeur

Université de Poitiers

Édition imprimée

Date de publication : 31 décembre 2006

Pagination : 203-223

ISBN : 978-2-911627-43-9

ISSN : 0765-0752

Référence électronique

Ilkay Südaş et Mustafa Mutluer, « Immigration européenne de retraités vers la « Riviera turque » : le cas d'Alanya (côte méditerranéenne) », Revue européenne des migrations internationales [En ligne], vol. 22 - n³ | 2006, mis en ligne le 31 décembre 2009, consulté le 15 avril 2022. URL : http:// journals.openedition.org/remi/3381; DOI : https://doi.org/10.4000/remi.3381

Ce document a été généré automatiquement le 15 avril 2022.

(c) Université de Poitiers 


\title{
Immigration européenne de retraités vers la « Riviera turque » : le cas d'Alanya (côte méditerranéenne)
}

\author{
Ilkay Südaş et Mustafa Mutluer \\ Traduction : Stéphane de Tapia
}

\section{Merci à Samim Akgönül pour sa relecture attentive.}

1 Les migrations du "troisième âge " vers les régions touristiques ont acquis une place nouvelle dans les migrations internationales et ce, plus particulièrement depuis environ vingt-cinq ans. Parmi les causes habituelles ou reconnues des migrations internationales, on retrouve les migrations de main-d'œuvre dues aux crises ou déséquilibres économiques ou la demande d'asile, donc de fait, la recherche d'un haut, ou au moins meilleur, niveau de sécurité et de prospérité. Dans cette optique, les migrations des personnes âgées liées au tourisme international occupent une place à part, que ce soit dans l'étude des causes ou dans celle des structures démographiques et économiques. Le désir de bénéficier au moment de la retraite d'un haut niveau de vie ou de meilleures conditions climatiques que dans la région d'origine joue ici un rôle non négligeable. Ces migrations se dirigent généralement du Nord vers le Sud ou, en d'autres termes, des régions plus froides vers les régions plus clémentes. Un numéro spécial de la revue International Journal of Population Geography (voir King et alii, 1998 : 109) montre bien cette tendance à une mobilité ou une migration croissante de personnes âgées de l'Europe septentrionale vers l'Europe méridionale. Williams et Hall (2000: 18) qualifient les personnes participant à cette migration liée au tourisme de "semi-retraités", "tournés vers la consommation», de personnes "passant leur vie entre deux domiciles ou plus ». Ils insistent sur le fait que ces migrations sont intimement liées aux structures démographiques des pays économiquement développés. Face à cette évolution, on remarque la difficulté de définir ces personnes, classées comme migrants par les uns, comme touristes par les autres. Il n'en reste pas moins que l'on peut 
considérer et classer ces personnes, qu'elles soient définies comme touristes ou immigrées, en fonction de leur statut juridique, leur statut de propriétaire et leur durée de séjour dans le pays d'immigration (Williams et Hall, 2000).

Dans la "littérature géographique » turque, les références sur ces mouvements de populations âgées des pays développés vers les pays aux climats plus cléments sont, pour notre pays, relativement nouvelles et encore limitées (Turan et Karakaya, 2005), bien que déjà évoquées dans le cas de quelques régions touristiques (Kaiser, 2003 ; Mutluer, 2004). Alors qu'au contraire, il est possible de relever de nombreux travaux concernant d'autres pays d'Europe, (King et alii; Warnes et Patterson; Willians et Patterson; King et Patterson; Rodriguez; Majoralas et Rojo (1998, Vol. 4, n 2) ou encore O'Reilly, 2000 et Kaiser 2001). Les travaux sur la Toscane (Italie), la Costa del Sol (Espagne) ou l'Algarve (Portugal) sont mieux connus ${ }^{1}$. On peut ajouter, parmi les dernières parutions en Tuquie, un ouvrage de Yllmaz (2005) traitant des Européens vivant à Istanbul, avec leurs réflexions personnelles et opinions à propos de la Turquie, "pays d'accueil et de résidence", qui nous renseigne sur cette nouvelle présence étrangère dans le pays, sujet relativement nouveau aussi pour les chercheurs locaux.

Depuis les années 1990, la Turquie aussi connaît cette importante migration dirigée des pays européens vers le Sud méditerranéen, formée de personnes d'âge mûr et, pour partie, retraitées. Les principales destinations de ces courants sont les régions touristiques des côtes méridionales et sud-occidentales du pays (mers Méditerranée et Egée). Kaiser (2003 : 271) situe leur installation au moment de la construction de logements dans les régions de villégiature de Bodrum (côte égéenne), de Marmaris ou d'Alanya (côte méditerranéenne). Il souligne la part croissante des originaires de l'Union européenne dans ces flux. Turan et Karakaya (2005) étudient quant à eux spécifiquement la présence britannique à Didim, non loin de Bodrum, sur la côte égéenne. Pour notre part, nous avons choisi de focaliser notre étude sur la présence dense des originaires d'Allemagne, des Pays-Bas ou des pays scandinaves (auxquels nous avons joint la Finlande par commodité) dans la ville d'Alanya, sur la côte méditerranéenne, non loin du grand pôle touristique d'Antalya. L'objectif de cet article est d'attirer l'attention sur la cible nouvelle que forme la section turque du bassin méditerranéen, en étudiant le cas de l'agglomération d'Alanya, en caractérisant cette migration européenne vers la Turquie, avec ses particularités et ses effets sur le tissu local.

\section{Le terrain de recherche}

4 Alanya est située sur la côte méditerranéenne de la Turquie, à 135 km à l'est d'Antalya, chef-lieu d'un département qui figure parmi les principales destinations touristiques du pays, internes comme internationales, avec environ 1000000 de touristes étrangers et dix millions de nuitées par an (Fig. 1 : localisation d'Alanya). Akova (1999) montre bien comment, avec $70 \mathrm{~km}$ de littoral et de plages, des conditions géographiques attrayantes (mer et montagne, sites historiques et plages) et un climat méditerranéen typique, la saison touristique peut durer jusqu'à sept à huit mois. Dans ces conditions, Alanya n'est plus une simple étape ou un espace touristique balnéaire, mais bien une région-cible, où s'installent durablement des étrangers et des migrants internes venus y passer une retraite agréable. L'arrondissement, tant pour le tourisme national que pour le marché international, a ainsi acquis une dimension nouvelle, par la mise en valeur de son 
patrimoine socio-culturel et naturel ; les touristes âgés venus d'Europe septentrionale et centrale y sont particulièrement réceptifs ${ }^{2}$.

Fig. 1 : Localisation d'Alanya

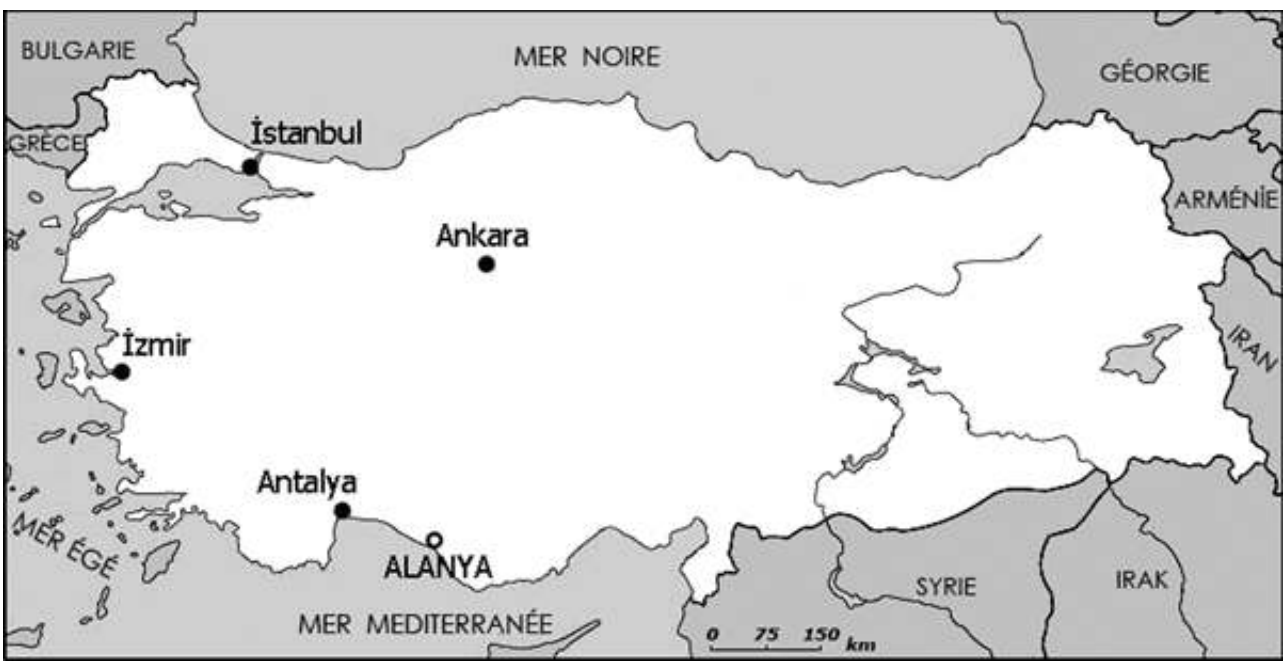

\section{Matériaux et données}

$\mathrm{Du}$ fait de la rareté des données statistiques et des références scientifiques sur un phénomène qui reste, nous insistons une fois encore, relativement nouveau en Turquie, il n'a guère été possible, de prime abord, de rassembler de données cohérentes sur la présence des "immigrés » européens à Alanya, au-delà de quelques renseignements donnés par les médias locaux. Nous avons donc commencé par la lecture de documents traitant des cas portugais, espagnols, italiens ou maltais pour établir une méthodologie adaptée à la situation qui nous intéressait.

6 L'étude repose sur une enquête spécifique sur deux terrains de recherche choisis dans l'agglomération. 118 personnes ont répondu à un questionnaire présenté lors de deux séjours d'un mois en juillet et novembre 2004, complété par des entretiens. Nos interlocuteurs étaient les représentants des autorités locales, des agents immobiliers, turcs et étrangers, des responsables de l'Église d'Alanya, des membres d'associations, du Conseil consultatif des Étrangers de la Municipalité d'Alanya et de la Direction locale de la Sécurité (services de Police). Ainsi ont été interviewés et/ou enquêtés : 72 ressortissants allemands, 24 Néerlandais, 12 Scandinaves, 4 Britanniques, 4 Autrichiens, 2 Irlandais et 31 agents immobiliers. Les questionnaires et les entretiens ont été menés en allemand (pour les ressortissants allemands et autrichiens), en anglais (pour les autres nationalités) et en turc (pour les acteurs locaux) ; nous avons utilisé le logiciel SPSS pour le traitement et la mise en forme des données.

\section{Les immigrés européens d'Alanya}

\section{Nombre et origines des immigrés}

7 Bien que la municipalité d'Alanya fasse état de la présence d'au moins 10000 Européens vivant sur son territoire sur son site Internet (www.alanya-bel.gov.tr), il est très 
difficile, sinon impossible de connaître ce chiffre avec certitude. Ainsi, selon la Direction de la Sécurité (Emniyet İlçe Müdürlüğ̈̈), 1633 ressortissants étrangers en possession d'un titre de séjour régulier - ceux que nous qualifions dans l'article de migrants ou immigrés, car possédant une résidence fixe - résidaient à Alanya en juillet 2004. Or, à la même date, on comptait 4062 logements construits et occupés par des étrangers, ce qui montre une présence étrangère, même provisoire, relativement importante, et nous approche des 10000 personnes estimées par les médias ou les acteurs locaux. Au premier rang des détenteurs de cartes de séjour figuraient les Allemands (62\%), suivis par les Scandinaves (20\%) et les Néerlandais (10\%), selon la Direction de la Sécurité de l'arrondissement d'Alanya (Alanya Emniyet ílçe Müdürlüğü, 2004). Les ressortissants des Iles britanniques (Anglais et Irlandais) suivaient avec seulement $5 \%$ des effectifs (Fig. 2: Origines des immigrés détenteurs de cartes de séjour).

Fig. 2 : Pays d'origine des personnes enquêtées

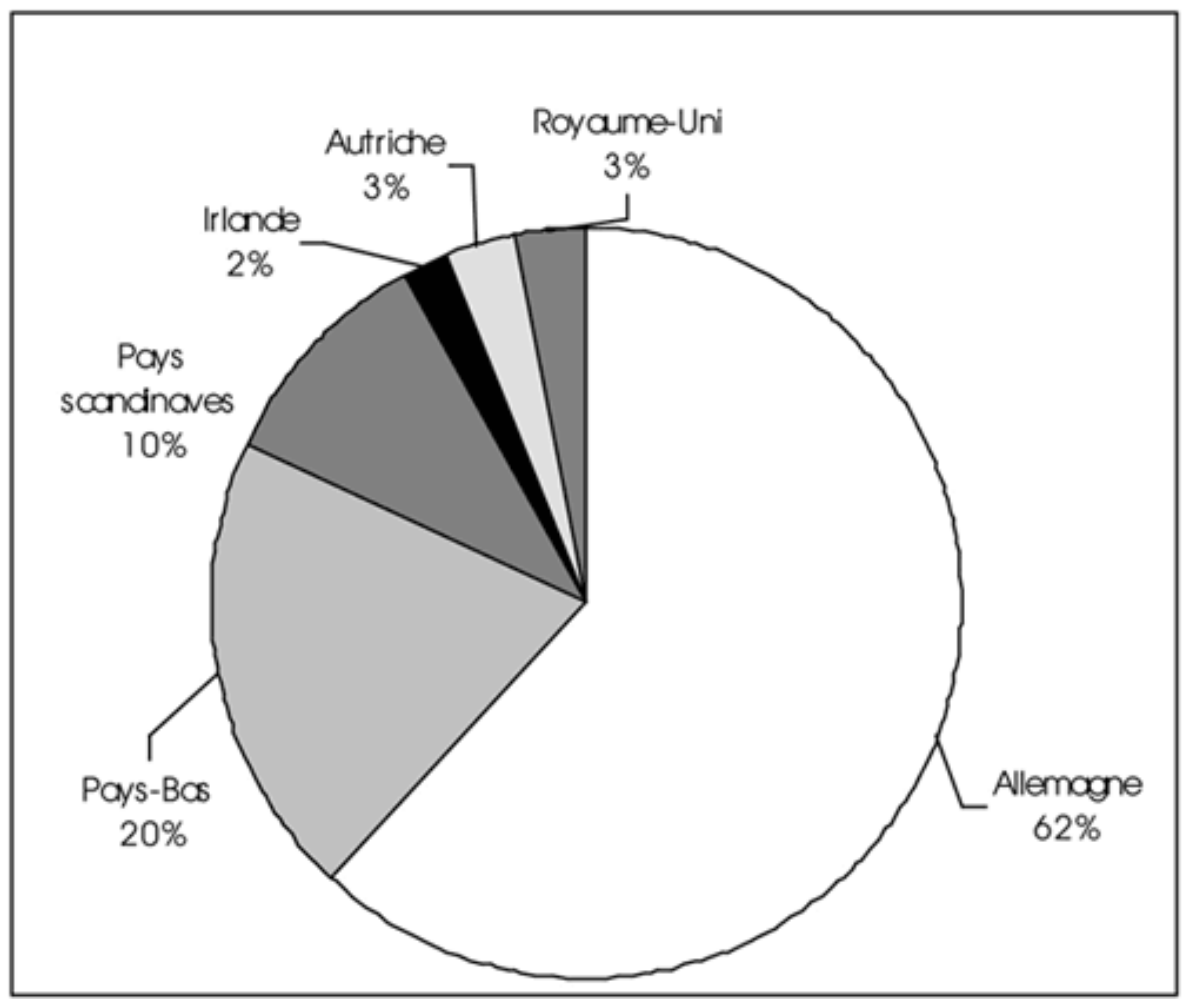

\section{Caractéristiques démographiques}

$59 \%$ des enquêtés sont des femmes pour $41 \%$ d'hommes. Si l'âge moyen des enquêtés est de 52 ans, près de la moitié ( $49 \%$ ) ont plus de 55 ans. Les plus jeunes forment des groupes plus limités. Ainsi, les $24-35$ ans ne forment que $8 \%$ de l'échantillon et les moins de 24 ans, seulement $3 \%$. La majorité de l'échantillon est formée par des migrants mariés $(60,2 \%)$. Les personnes célibataires, divorcées ou ayant d'autres statuts maritaux (veufs et veuves, concubins ou couples non officiels) sont donc moins nombreuses. $38 \%$ seulement des enquêtés sont réellement retraités. Si l'on se base sur ces données partielles, on peut donc parler d'une migration de personnes relativement âgées à l'échelle de la Turquie, mais assez jeunes selon les normes européennes ${ }^{3}$. On 
voit donc que la majorité des migrants est composée de couples mariés (ou statuts assimilables) et non de personnes isolées.

Le niveau scolaire de ces migrants est relativement élevé : $22 \%$ des enquêtés ont acquis un diplôme universitaire, la plupart $(70 \%)$ ayant un niveau secondaire: lycée professionnel, lycée classique ou cours professionnels. Les statuts professionnels déclarés sont diversifiés : le groupe étudié comprend des métiers artisanaux (coiffeur, plombier, ébéniste, boulanger...), des fonctionnaires et employés, des femmes au foyer (9\%) et divers métiers liés à la vente et au commerce. On peut remarquer que 8,5\% des enquêtés n'ont pas donné de définition claire ou satisfaisante à cette question. Les questions sur les niveaux de revenus, les dépenses ou le niveau d'épargne n'ont malheureusement pas plus fourni de données satisfaisantes et n'ont pu caractériser de groupes économiques pertinents. Ainsi, à chaque fois que la question a été évoquée par un enquêteur, pour près de la moitié de l'échantillon ( $44,1 \%)$, l'enquêté préférait ne pas répondre, arguant qu'il s'agissait d'une information d'ordre privé. On peut comparer cette situation à celle de l'enquête effectuée par Turan et Karakaya (2005) qui ont obtenu les mêmes réponses de la part des Anglais interrogés à Didim. Il semble cependant que le niveau de revenu déclaré soit le plus souvent situé entre 1000 et $2500 €$ mensuels.

10 La plupart des migrants demeurent à Alanya en renouvelant, soit leur carte de séjour (oturum tezkeresi, oturum izini), soit leur visa touristique. Les visas de tourisme renouvelés comptent pour $70 \%$ des effectifs enquêtés, les visas de tourisme nouveaux pour $22 \%$, donnant ainsi un statut légal, mais précaire pour $92 \%$ du total des Européens résidant à Alanya. De fait, la différence est établie selon la durée de séjour légal. Un petit groupe se détache, s'agissant d'étrangers mariés avec des ressortissants turcs et possédant un statut stable de conjoints de nationaux turcs ( $7 \%$, cf. tableau 1).

Tableau 1 : Statut juridique des immigrés d'Alanya - Turquie

\begin{tabular}{|l|l|}
\hline Type de Statut & $\%$ \\
\hline Je renouvelle mon visa périodiquement & 70 \\
\hline Je suis touriste & 22 \\
\hline J'ai (également) la nationalité turque & 7 \\
\hline Non déterminé & 1 \\
\hline
\end{tabular}

Source : Enquête des auteurs, 2004.

\section{Facteurs de migration vers Alanya}

11 Pour les immigrés européens, le facteur premier de choix de séjour à Alanya est sans conteste le climat méditerranéen. À l'inverse, il semble bien que le facteur répulsif du côté du pays d'origine soit justement le climat océanique ou continental européen. Les Européens ayant migré vers Alanya évoquent leur volonté d'échapper aux conditions climatiques, décrites comme froides et humides, de leur pays. Lorsqu'ils comparent les éléments du climat local avec la situation de leur ville d'origine, les immigrés sont très 
précis. La température moyenne et saisonnière est en effet un argument majeur de la décision de s'installer en Turquie, ne serait-ce que quelques mois dans l'année. Ainsi, on peut remarquer que la température moyenne maximale enregistrée en janvier à Narvik (Norvège), lieu d'origine du répondant le plus septentrional de notre échantillon, est de $1,9{ }^{\circ} \mathrm{C}$., qu'elle est de $-0,9{ }^{\circ} \mathrm{C}$. à Stockholm, de $1,1^{\circ} \mathrm{C}$. à Munich alors qu'elle atteint $16,2{ }^{\circ} \mathrm{C}$. à Alanya au même moment. De même, les températures moyennes minimales enregistrées sont de $-7{ }^{\circ} \mathrm{C}$. (Narvik), $-5,1^{\circ} \mathrm{C}$. (Stockholm), $-5,7{ }^{\circ} \mathrm{C}$. (Munich) contre $8,4^{\circ} \mathrm{C}$. à Alanya (Müller 1980). On peut donc parler de «fuite vers le soleil » pour ces habitants du nord de l'Europe, comme une des raisons de la migration vers la Méditerranée orientale. À l'inverse, on observe un mouvement contraire de certains des répondants - l'enquête a été effectuée en deux temps, juillet et novembre qui préfèrent quitter la région d'Alanya en raison des $40^{\circ} \mathrm{C}$. qu'enregistre souvent la côte méditerranéenne en juillet et rentrer dans leur pays ou leur région d'origine pendant les mois les plus chauds de l'été. Pour ces personnes habituées au froid hivernal et à la douceur estivale, la chaleur lourde et étouffante de la côte méditerranéenne turque, avec un fort taux d'hygrométrie, est difficilement supportable. Alors qu'à Alanya les hivers sont doux et les saisons intermédiaires, printemps et automne, sont chaudes et ensoleillées mais très supportables, on assiste en fait à une migration saisonnière entre deux pôles : les mois d'été en saison chaude des régions de départ, le reste de l'année en Turquie, soit de fait la majeure partie de l'année.

12 Le second facteur attractif de la région est manifestement le coût de la vie. La Turquie garde une image de pays peu cher dans une grande partie du monde et en particulier dans beaucoup de pays européens. Özgüç (2003:223) fait remarquer que les touristes internationaux, toutes choses égales par ailleurs, hésitent à partir durablement vers des pays jugés trop chers pour leurs niveaux de revenus. Ainsi, si les deux conditions principales, climat favorable et coût de la vie, sont réunies dans les destinations touristiques internationales certains préfèreront au-delà de premiers courts séjours touristiques de découverte et d'adaptation, transférer leur domicile, au moins pour une partie de l'année. Plutôt que de passer une retraite à l'étroit avec un revenu très moyen, certains ressortissants de l'Union européenne préfèrent vivre en Turquie avec une retraite qui les met au niveau de catégories sociales turques plus aisées. Presque la moitié ( $46 \%$ ) des enquêtés ont indiqué que leur décision est liée à l'aspect financier.

Les migrants mettent également en avant la qualité de vie rencontrée en Turquie, en particulier la chaleur des rapports humains, la facilité de nouer des contacts, les relations amicales de voisinage, l'intérêt que les habitants se portent, en contraste avec la froideur et l'anonymat des relations sociales et humaines des régions quittées, définitivement ou périodiquement. La mise en exergue de l'hospitalité traditionnelle turque par les services officiels d'information semble avoir ici porté ses fruits, mais cela ne suffit sans doute pas à expliquer l'engouement montré par les migrants. C'est pourtant le cas à Alanya où les migrations de ce type semblent vérifier ce slogan touristique. $40 \%$ des enquêtes citent l'hospitalité turque et la chaleur des relations humaines comme facteurs d'attractivité les ayant persuadé de s'installer à Alanya et $45 \%$ des personnes interrogées mettent en avant la froideur et la pauvreté des relations humaines dans leur propre pays comme facteur répulsif ayant entraîné leur décision de partir vers le Sud. $13 \%$ de l'échantillon insiste même sur la solitude éprouvée dans le pays d'origine comme raison de départ. Les enquêtés soulignent la 
froideur de leurs compatriotes, le manque d'intérêt ressenti, la distance entre les individus. Si l'on agglomère carences ressenties dans les relations humaines et sentiment de solitude, la part des individus se déclarant insatisfait des relations humaines dans leur région d'origine est de $58 \%$. Cette insatisfaction générale a motivé une réelle désaffection et une fuite vers des climats jugés plus chaleureux dans tous les sens du terme.

L'environnement naturel est cité comme troisième facteur d'attractivité de la région : $43,2 \%$ des enquêtés le mettent parmi les raisons de s'installer dans la région. Le « trinôme mer-sable-soleil » de la côte méditerranéenne n'est pas le seul cité puisqu'on insiste aussi sur les paysages de montagne, la richesse du patrimoine naturel avec ses grottes et ses fleuves côtiers, la possibilité de safari dans l'arrière-pays des montagnes du Taurus ${ }^{5}$.

Le mariage et le travail font partie des raisons invoquées pour la migration vers Alanya. Le mariage, car il est indiqué par $22 \%$ de notre échantillon. Ce facteur peut être renforcé du fait que $81 \%$ de nos enquêtés sont des femmes, toutes nationalités confondues. Le travail est invoqué par $8,5 \%$ de l'échantillon et c'est peut-être là le facteur le plus surprenant. Cela peut pourtant s'expliquer par le fait que la majorité des personnes rencontrées sont des retraités ou des individus proches de la retraite, parfois inactifs. C'est ainsi que ce petit groupe de travailleurs (entre 30 et 52 ans) a investi le secteur de l'immobilier et du tourisme, assuré de trouver un créneau commercial et de rapport intéressant, avec une forte plus-value.

On comparera ci-dessous (figures 3 et 4 ) les facteurs attractifs et répulsifs ayant entraîné la décision de partir pour s'installer en Turquie et plus particulièrement à Alanya.

Fig 3: Facteurs attractifs selon l'enquête

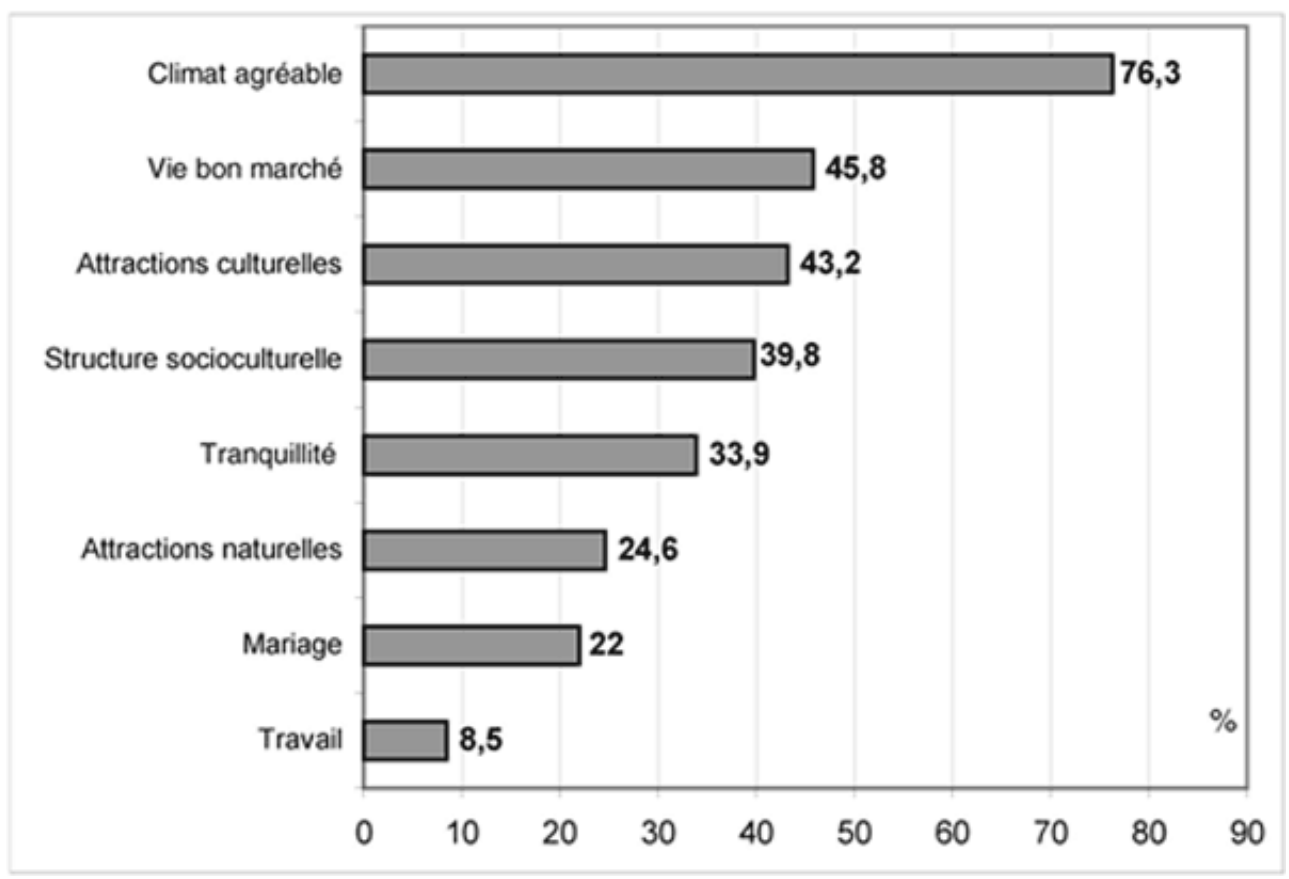


Fig. 4: Facteurs répulsifs selon l'enquête

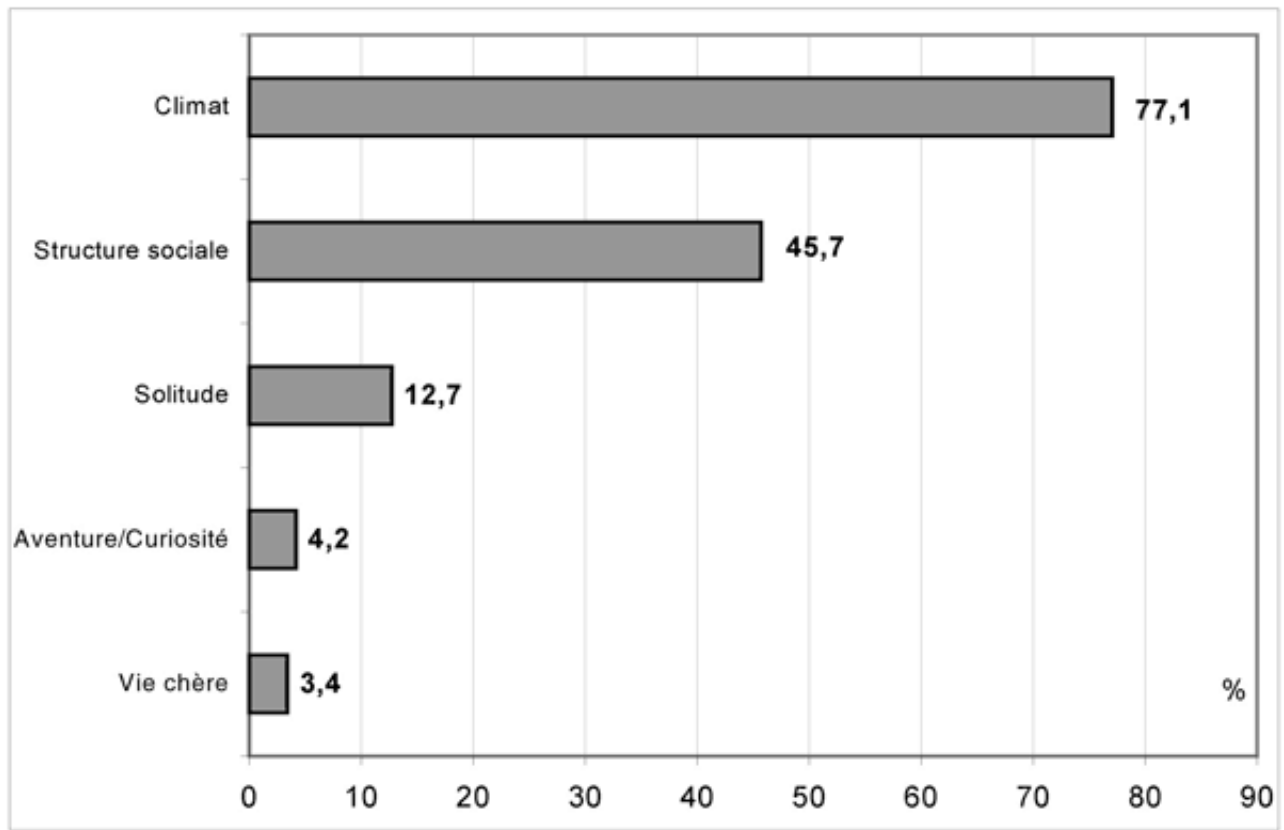

\section{Impacts de la migration des Européens sur Alanya} internationales de retraités ont des conséquences importantes dans les domaines économiques, culturels, sociaux et politiques. La tendance va dans le sens d'une augmentation de la population résidente et d'une demande accrue de logement. On observe une accélération de la croissance urbaine et une augmentation de la consommation de personnes retraitées disposant de revenus supérieurs à la moyenne locale, ce qui en définitive assure des revenus considérables à la région d'immigration. Nazmiye Özgüç écrivait déjà en 1977, en insistant sur la présence de migrants retraités dans les régions touristiques et sur leurs effets: "Les maisons de vacances estivales construites en particulier dans des régions au climat agréable, avec l'abaissement de l'âge de la retraite, finissent par devenir des logements permanents appartenant aux retraités dont le nombre augmente. Par exemple, la Floride et la Californie, les côtes méditerranéennes comme la Côte d'Azur, sont devenues des régions de grande concentration des retraités. C'est ainsi que les structures démographiques et urbaines en sont durablement affectées. » (Özgüç, 1977 : 152).

Les régions-cibles de la migration de retraités et personnes âgées connaissent un transfert et une mutation culturels non négligeables. Ainsi, ces migrations de retraités jouent un rôle important dans la construction des paysages culturels (cultural landscape). Ce changement lié à la culture des migrants mérite d'être souligné en Turquie. La Turquie est en effet à la fois pays d'émigration (depuis les années 1960) et pays d'immigration (progressivement, depuis les années 1990) et commence tout juste à connaître des mutations déjà observées depuis longtemps ailleurs, par exemple entre l'Europe du nord et l'Europe méditerranéenne ou entre le Canada et les États-Unis. Mais les différences économiques et culturelles entre la Turquie, nouveau pays de destination de la migration de retraités, et les pays d'origine d'Europe occidentale conduisent à interroger la nature des mutations observées. Dans cette relation nouvelle 
quels sont les changements observés à Alanya? Parmi les effets les plus visibles figurent l'explosion du marché immobilier régional lié à la présence étrangère (déjà fortement modifié par la demande nationale du tourisme intérieur) et les impacts sur le paysage culturel local et urbain.

\section{Effets sur le marché immobilier et l'urbanisation de l'agglomération}

19 Comme le remarquent Turan et Karakaya (2005) à propos de la présence britannique à Didim, les migrations de retraités amènent un pouvoir d'achat de nature à vivifier le marché local et l'économie générale de la région concernée (biens de consommation courante, loisirs, restauration, services à la personne...); ce sont surtout le marché immobilier et le commerce de détail qui profitent de ce nouvel apport, mais tous les secteurs économiques en retirent aussi un bénéfice. Ainsi, dans l'exemple d'Alanya, Armutçu (2004) estime que le chiffre d'affaires du seul secteur immobilier atteint les 2 millions d'Euros. La visibilité et la vitalité de ce secteur d'activité sont frappantes dans toute la ville et ses abords immédiats. Ainsi le nombre d'achats de biens immobiliers par des étrangers est passé d'environ 250 en 1997 à 300 en 1998, mais connaît une accélération dans les années 2000 ; il a été de plus de 800 sur les premiers neuf mois de l'année 2004 (Fig. 5).

L'enquête auprès des agents immobiliers montre que ce développement du marché dans l'ensemble de l'arrondissement est bien lié à l'immigration. Les agents propriétaires de leur fonds de commerce sont pour seulement $23 \%$ d'entre eux des originaires d'Alanya. La moitié des agents rencontrés, originaires d'autres régions, disent que le travail et le tourisme en tant que gisement d'emploi sont la cause de leur venue à Alanya. Pour $75 \%$ d'entre eux, le secteur immobilier est une nouvelle occupation (après un autre emploi ou une autre affaire ailleurs). C'est bien l'opportunité créée par ce nouveau marché et la densité de la demande qui les ont attirés ici. On remarque aussi que $35 \%$ des agents et de leurs employés ne sont pas de nationalité turque! On remarque que l'année 2000 est l'année du déclenchement du boom immobilier, au moment même où arrivaient de nombreux étrangers intéressés par un achat et une installation dans la région. Si le véritable engouement des étrangers pour l'immobilier, et les ouvertures d'agences se situent autour de l'année 2000 nos données indiquent cependant que cette période a été précédée d'une première découverte du milieu par les futurs migrants, dès le milieu des années 1990 (comparaison des Fig. 5, 6 et 7). 
Fig. 5 : Nombre d'étrangers ayant acquis des biens immobiliers à Alanya, de 1997 à 2004

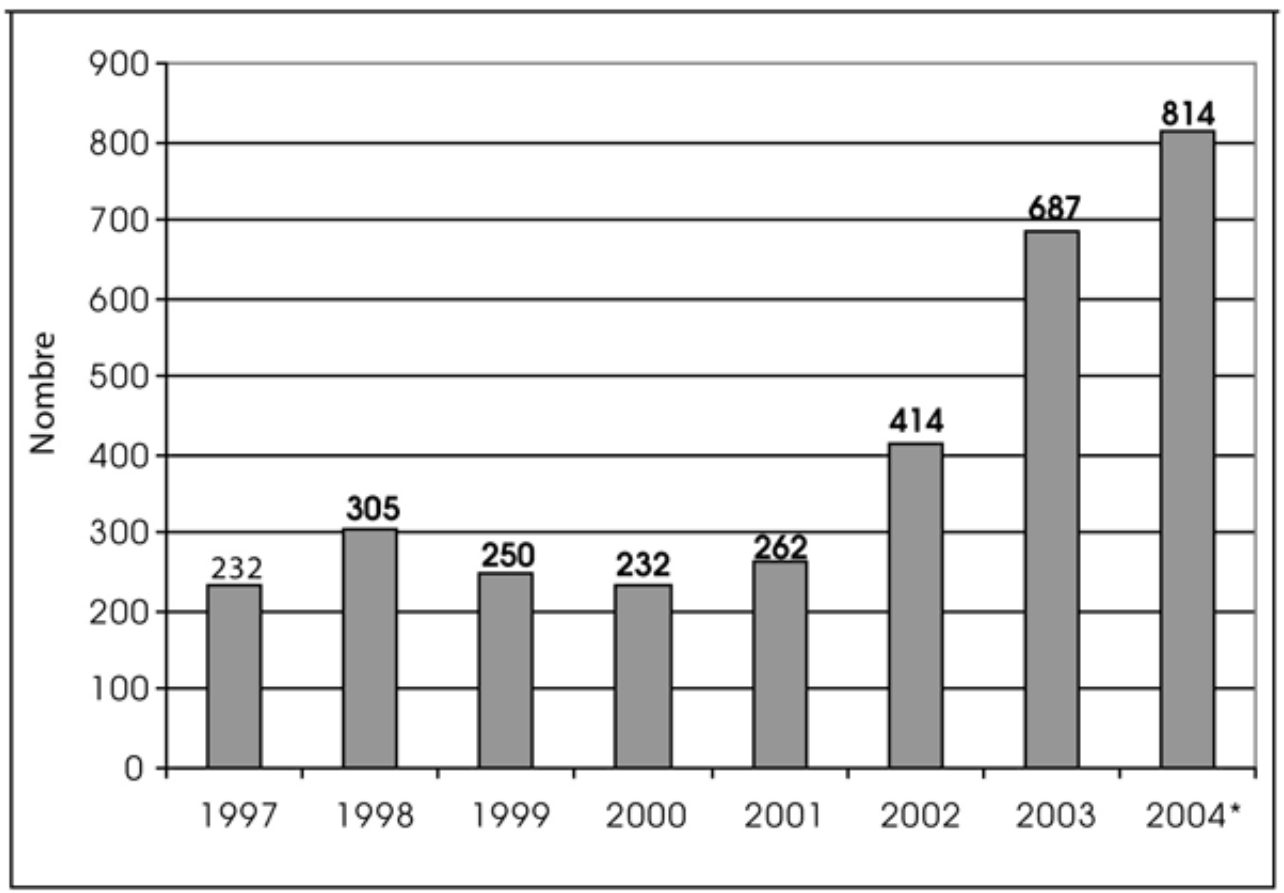

Fig. 6 : Période d'achat de biens par les répondants à l'enquête

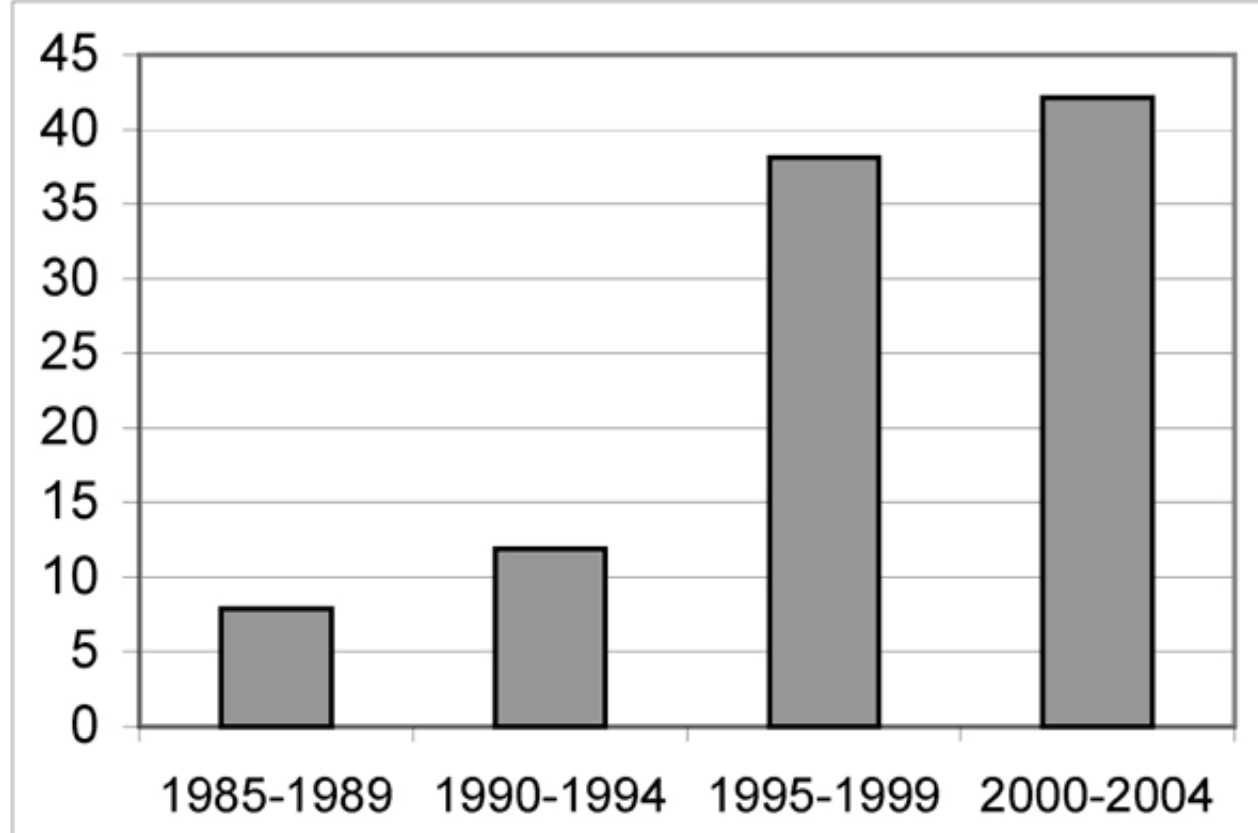


Fig. 7 : Périodes d'ouverture des nouvelles agences immobilières

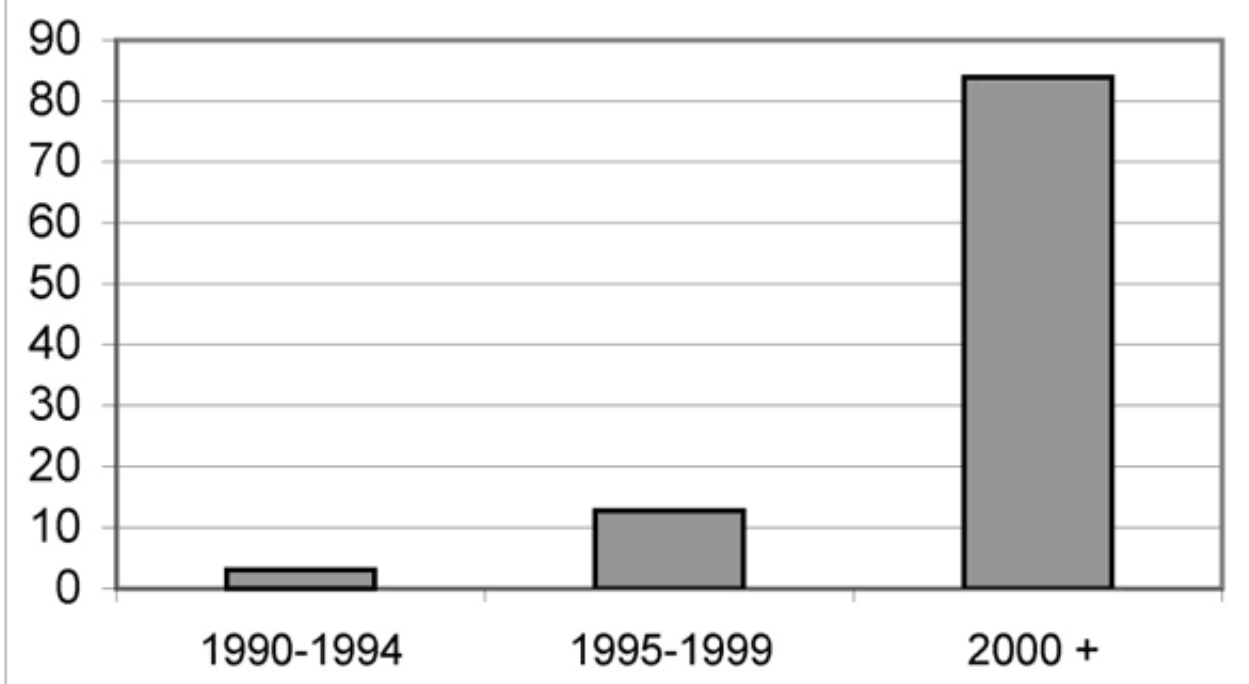

Dans ce marché, actif mais passablement déséquilibré en faveur des Européens, les ressortissants turcs n'arrivent qu'en quatrième position, derrière, en ordre d'importance, les acheteurs allemands, néerlandais et scandinaves. Ces dernières années, on observe l'apparition sur ce marché d'acheteurs belges et irlandais. Répondant à cet état de fait, il n'est pas rare que les panneaux publicitaires ou d'information soient directement rédigés dans une langue autre que le turc ou en plusieurs langues étrangères (cf. illustration $n^{\circ} 1$ : panneaux et devanture de l'agence en allemand).

Touchée par l'immobilier touristique, l'actuelle agglomération d'Alanya montre une nette tendance à un développement linéaire le long de la côte et de la route côtière (RN 400), en direction de l'est (vers Anamur et Mersin) et de l'ouest (vers Antalya). Partant du centre-ville dans les deux directions opposées, on observe la mise en chantier et la construction dans toutes les localités côtières non classées en municipalités (belde) de cités balnéaires de résidences secondaires (yazlk siteleri), de quartiers de villas ou de résidences collectives ${ }^{6}$. Si l'on prend en compte la demande en logements nouveaux, qu'il s'agisse de migrants ou de résidents étrangers originaires d'Europe, de touristes intérieurs ou de personnes vivant sur place, on voit combien ce marché de la construction a pris une place importante dans l'économie locale. Mais si l'on peut dire que la présence étrangère n'a pas pour l'instant affecté sérieusement la démographie locale, on peut néanmoins se poser la question pour les années à venir (attraction de migration interne liée à l'emploi, vieillissement de la population, besoins nouveaux de cette population). De fait, les 1633 immigrés déclarés (en possession d'un permis de séjour) ne représentent que $2 \%$ des 88346 habitants du chef-lieu d'arrondissement (ville d'Alanya au recensement de 2000), mais on peut citer le cas de Kestel avec ses 5 623 habitants permanents recensés en 2004, où 645 Européens ont acquis une propriété immobilière, soient au moins 645 ménages étrangers ou environ $12 \%$ de la population. À n'en pas douter, la pression immobilière continuera à s'exercer pendant les années qui viennent et la structure démographique d'Alanya en sera affectée. 


\section{Effets des nouveaux immigrés sur le paysage culturel urbain}

23 traditionnelle confrontée à un tourisme de masse incluant de nombreux étrangers qui décident de s'installer durablement. Le paysage géographique et culturel urbain change avec la venue des Européens. On rencontre ici deux types de diffusion du changement : diffusion par saut ou diffusion par élargissement d'un phénomène spatialisé. Le premier type semble être le plus courant: il s'agit d'opportunités foncières ou immobilières permettant la création d'un îlot d'habitat, d'une résidence touristique, sans continuité avec le bâti existant, dans un contexte où la planification urbaine et le zonage font défauts ou ne sont pas respectés. Dans le second cas, il y a continuité spatiale du processus de diffusion de la présence étrangère.

Le centre-ville d'Alanya a connu des changements de physionomie par l'implantation d'activités très spatialisées. Les cafés-bars - lieux de rencontre des Européens -, sont en particulier fréquentés par les Allemands et les Néerlandais. Ces espaces sont à la fois un endroit de socialisation pour les nouveaux venus, où l'on se sent chez soi et entre soi et où l'on échange conseils pratiques et informations sur Alanya et les environs. On peut aussi partager les expériences et les problèmes rencontrés, phénomène classique de toutes les migrations internationales. En plus de ces lieux de rencontre, on trouve des bureaux de presse en langue étrangère (au moins trois journaux édités en allemand, voir Fig. 8 : plan d'Alanya), des locaux associatifs, des bureaux privés d'information, voire des lieux de culte chrétiens. L'emploi d'une ou plusieurs langues non turques est fréquent (panneaux d'information, panneaux publicitaires sont le plus souvent rédigés en allemand et en anglais). C'est un fait nouveau en Turquie, d'autant plus remarquable qu'il est apparu très récemment. Ces espaces de marquage de la présence européenne sont plus fréquents dans le quartier dit « du Marché » (Çarsı Mahallesi), au centre-ville commercial, près du port de plaisance et sous la forteresse byzantine et seldjoukide (Fig. 8) $)^{7}$.

Les faits culturels les plus saillants, dans un pays centralisé et officiellement monolingue comme la Turquie, sont les usages linguistiques et religieux des Européens à Alanya. En raison de la demande des Chrétiens vivant dans l'arrondissement, la Municipalité a accordé le droit d'utiliser une salle du Centre Culturel municipal (Alanya Belediyesi Kültür Merkezi) comme lieu de prière; un cimetière chrétien a même été aménagé à côté du cimetière musulman (illustration $n^{\circ} 2$ ). Un prêtre " œcuménique » est mis à la disposition des Chrétiens pratiquants par le Consulat d'Allemagne, mais des démarches sont en cours pour l'ouverture d'une paroisse propre aux migrants ${ }^{8}$.

Quant au marquage linguistique, il est frappant de constater qu'il se généralise dans toute la ville; la quasi-totalité des plaques commerciales et des publicités est rédigée dans une langue étrangère. Même la Municipalité a jugé bon de se doter d'une plaque trilingue très officielle portant les mentions «BELED İYE », « RATHAUS », «TOWNHALL » (Illustration $n^{\circ} 3$ ). L'édition en langues étrangères de bulletins locaux (allemand et anglais) est un autre phénomène digne d'intérêt dans le contexte turc. Édités et imprimés directement au centre-ville, ces journaux et bulletins sont diffusés sur toute la côte et sont un bon exemple de cette diffusion culturelle. Par ailleurs, on remarque que tous les prix sont affichés par les commerçants locaux en Euros et non en YTL (Yeni Türk Lirası : nouvelle livre turque)! D'autre part, le processus de « diffusion par saut » peut être illustré par l'exemple de la boulangerie allemande et plus encore 
par l'apparition d'une boucherie allemande vendant de la viande de porc $^{9}$ (Illustrations $\mathrm{n}^{\circ} 4$ et 5). Rappelons que la Turquie, pays laïque, est malgré tout massivement musulmane et que le gouvernement élu est de sensibilité islamiste. L'ouverture de ce genre de commerce est une petite révolution.

Fig. 8 : Les principales implatations de services destinés aux immigrés européens dans la ville d'Alanya

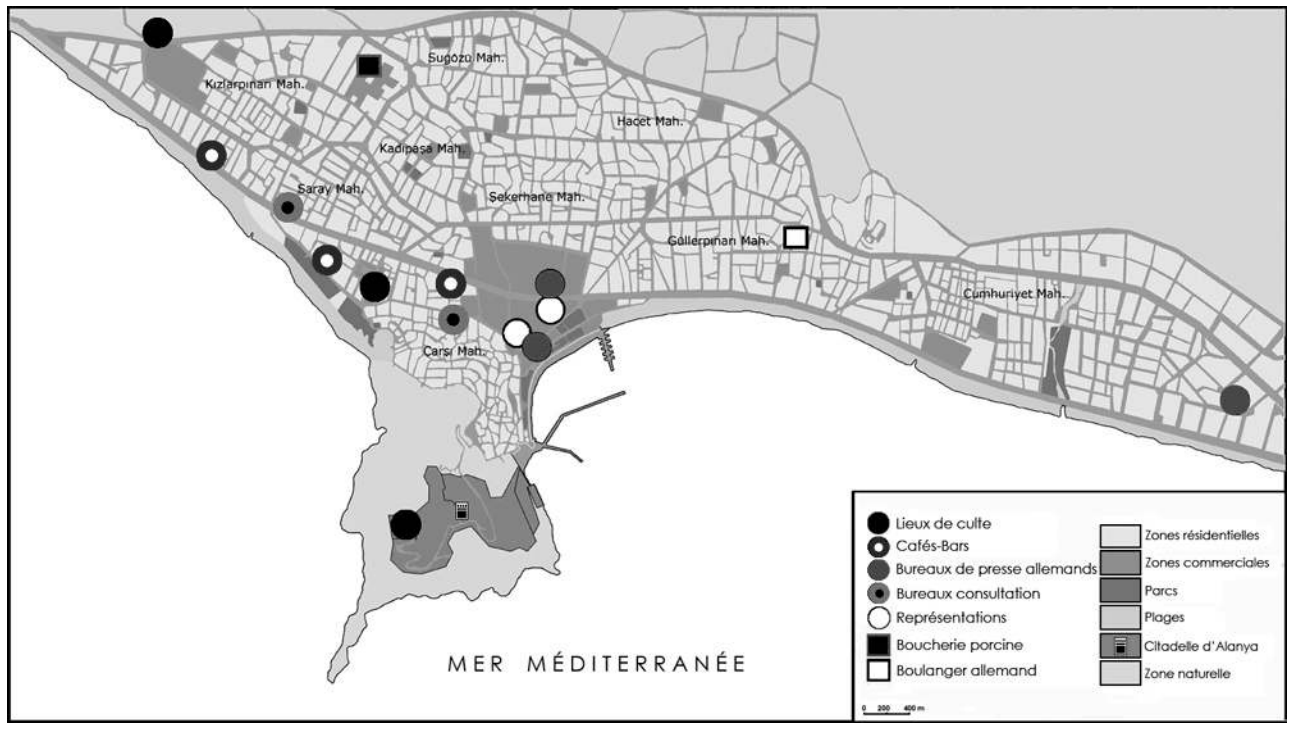

\section{Conditions de vie des immigrés et problèmes rencontrés}

La présence étrangère et l'existence de populations européennes dans les agglomérations touristiques des côtes occidentales (égéennes) et méridionales (méditerranéennes) de Turquie ont souvent été décrites par les médias, avant que les chercheurs en sciences sociales ne s'y intéressent, comme le remarque O'Reilly (2000: 2) à propos de la présence, puis de l'immigration de retraités britanniques sur les côtes espagnoles. Dans les années 1980, c'est souvent la presse qui a attiré l'attention des pouvoirs publics sur la présence anglaise en multipliant les articles de presse, les reportages radiophoniques et télévisés. La Turquie montre des faits comparables. Ce sont d'abord les journalistes allemands du magazine der Spiegel ou la version turque du magazine américain National Geographic (par exemple les articles d'A. Hielscher en 2003 ou d'E. Armutçu) qui ont décrit les conditions de vie et d'installation de ces migrants d'un type nouveau pour la Turquie. Les grands quotidiens ont fait à leur tour des reportages, mais en mettant l'accent sur le marché immobilier, à l'instar du quotidien Hürriyet (le 21.01.2005) ou de Radikal le (07.07.2003).

Ces immigrés sont avant tout des touristes et ils mènent leur vie dans un environnement de vacances et de loisirs. Ainsi se promener et voyager, faire des achats, pratiquer un sport, s'amuser, passer du temps à jardiner sont parmi les principales activités exercées par les migrants (Tableau 2).

Tableau 2 : Le temps libre des enquêtés

\begin{tabular}{|l|l|l|}
\hline Activité pratiquée & Fréquence & $\%$ \\
\hline
\end{tabular}




\begin{tabular}{|l|l|l|}
\hline Promenade, tourisme & 77 & 65,3 \\
\hline Faire des emplettes & 72 & 61,0 \\
\hline Pratique d'un sport & 33 & 28,0 \\
\hline Loisirs divers & 29 & 24,6 \\
\hline Entraide, bienfaisance & 23 & 19,5 \\
\hline Jardinage & 18 & 15,3 \\
\hline
\end{tabular}

Source : Enquête des auteurs, 2004.

Alanya a acquis une réputation de centre immobilier où les affaires sont plutôt faciles et de rapport financier rapide. Certains des migrants travaillent dans des associations d'entraide et de solidarité, par exemple dans la protection et la stérilisation des animaux errants (activité surprenante pour beaucoup de Turcs, rarement choqués de voir des chats ou des chiens errants, tout en les craignant d'ailleurs) ou plus sérieusement en animant des campagnes de soutien aux élèves de familles pauvres. Mais on trouve aussi des personnes s'investissant dans le Conseil consultatif des Étrangers, ou dans l'édition de la presse locale.

Concernant les problèmes cités par les migrants, ils peuvent être classés en deux catégories: une première catégorie, environ la moitié de notre échantillon, relève divers problèmes et s'en plaint, l'autre moitié dit ne pas rencontrer de problèmes du tout dans la vie quotidienne. Les "plaignants » citent dans l'ordre la bureaucratie turque, l'absence de pratique de la langue turque, les droits insuffisants reconnus aux étrangers en Turquie et la difficulté de la pratique religieuse (Fig. 9). Plus de $30 \%$ des répondants se plaignent de la complication et des lenteurs des démarches administratives en Turquie, de l'absence d'informations fiables et de service d'orientation sur le statut des étrangers. La difficulté de communiquer en turc ou dans d'autres langues avec les administrations locales prend tout son sens si l'on connaît les arcanes de la bureaucratie turque. En d'autres termes, plus que la langue proprement dite, c'est la pratique administrative qui se révèle un réel problème. Ces nouveaux résidents d'Alanya connaissent des difficultés pour acheter leur maison, payer leurs factures d'électricité ou de téléphone, solutionner des questions juridiques, communiquer avec les autorités locales, obtenir leur carte de séjour à la direction locale de la Sécurité, bref à chaque étape du processus d'installation. Il est assez piquant de constater que les Européens de Turquie se plaignent souvent de difficultés que connaissent trop souvent les Turcs en Europe. 
Fig. 9 : Problèmes ressentis par les enquêtés à Alanya

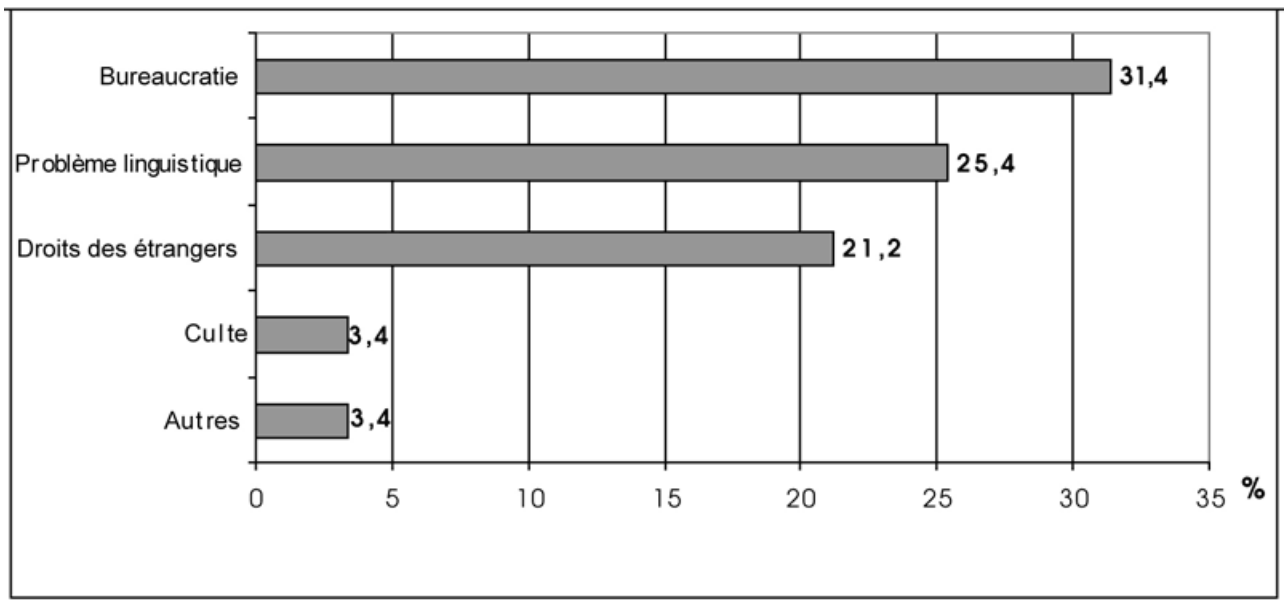

Dans un proche avenir, on peut penser qu'il sera difficile de régler ces problèmes en multipliant les interprètes ou les brochures en plusieurs langues. Mais si $34 \%$ des répondants affirment ne connaitre que quelques mots de turc et $7 \%$ ne rien savoir en turc, si seulement $2,5 \%$ disent le parler correctement, le nombre de non-locuteurs absolus n'est dans notre enquête que de 8 sur 118 ! (Fig. 10). Dans la mesure où nombre de commerçants et d'agents immobiliers locaux maîtrisent plus ou moins les principales langues européennes et où les Européens immigrés à Alanya commencent à s'organiser culturellement, on peut penser que cet obstacle va aller en s'amenuisant, les deux populations trouvant intérêt à communiquer.

Fig.10 : Niveaux de connaissance du turc selon les enquêtes

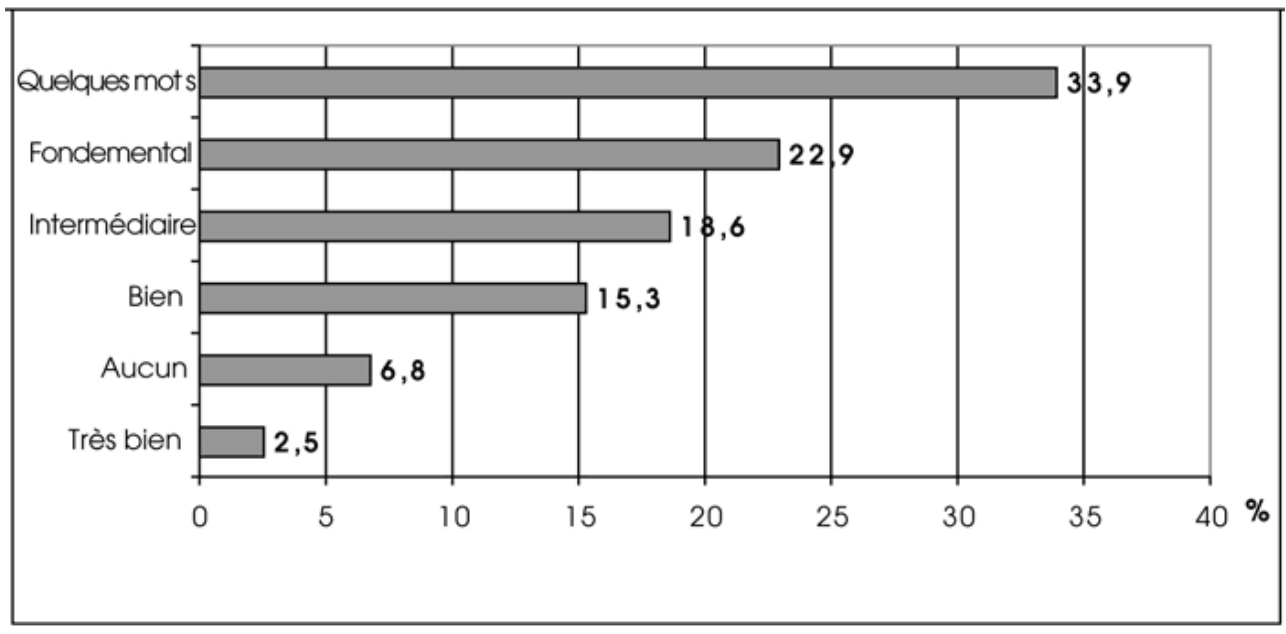

Afin de répondre à la demande de la population chrétienne dans l'arrondissement, la Municipalité d'Alanya a affecté une partie d'une ancienne église historique transformée en centre culturel municipal (Alanya Belediyesi Kültür Merkezi) au culte, mais certains migrants continuent cependant à revendiquer l'ouverture d'un véritable lieu de culte. Quant aux droits reconnus aux migrants étrangers, les permis de séjour et de travail restent objets de plaintes. L'octroi par l'administration d'un droit au travail passe par un long et pénible chemin bureaucratique : en Turquie, un étranger peut travailler sans problème dans les secteurs non interdits aux étrangers, mais pour l'obtention d'un permis de travail, il lui faut demander un visa de travail (çalışma vizesi), puis demander 
une permission de travailler (çalışma izni) qui impose l'obligation d'une carte de séjour (ikamet tezkeresi). On observe ici, comme dans le cas de la possession par des étrangers de biens immobiliers, une clause de réciprocité (Aybay 2005 : 152). Quant aux autres problèmes rencontrés, on signale la difficulté de se repérer dans les prix pratiqués (en d'autres termes, l'existence d'escroqueries), les coupures d'électricité, la difficulté d'obtenir par l'administration les plaques d'immatriculation pour les véhicules, les chiens et chats errants dans les rues, l'absence d'aménagement urbain et le bruit ${ }^{10}$.

Un Conseil (consultatif) des Étrangers (Yabancilar Meclisi), composé de membres de diverses nationalités, se trouve aux côtés de la Municipalité d'Alanya (Alanya Belediyesi) pour faire remonter les problèmes rencontrés par les étrangers et chercher avec les autorités locales des solutions à ces problèmes. La simple présence de ce conseil est un signe important de la volonté d'intégrer ces Européens à la vie locale en acceptant leur présence et en cherchant à leur faciliter les conditions d'existence, c'est aussi montrer que ces Européens font maintenant partie d'Alanya. Dans la résolution de ces problèmes, d'autres alternatives se font jour, comme l'existence de sociétés privées de services et d'information tournées vers la population étrangère. De plus, Alanya a vu la création de deux associations composées de résidents allemands, jouant un rôle important dans la vie sociale de la petite communauté.

\section{Conclusion}

On trouve encore assez peu de littérature spécialisée sur la migration de retraités en Turquie, fondée sur des données statistiques suffisantes et pourtant le phénomène de la migration internationale de retraités européens, originaires surtout du Nord et du nord-Ouest du continent, prend de l'ampleur. L'intérêt pour cette forme de migration est aussi plus récent que dans d'autres pays méditerranéens. La Turquie se trouve dans une phase de développement de cette forme migratoire, apparue dans les années 1990 et qui s'est véritablement accentuée dans les années 2000. Alors que ce processus est bien connu sur les côtes et dans les îles de l'Espagne et dans d'autres pays riverains de la Méditerranée comme le Maroc, il n'en est qu'à ces débuts sur les côtes turques.

Alanya, agglomération-témoin exemplaire, a permis de repérer les caractéristiques des migrations internationales dirigées vers les régions touristiques de Turquie. Elle a vu des migrants européens, principalement allemands, néerlandais et scandinaves, s'implanter en nombre important. Si l'on se réfère à l'âge moyen de ces migrants, on peut parler d'une migration du " troisième âge " selon les critères turcs où l'espérance de vie est bien moindre qu'ailleurs en Europe. Mais dans la mesure où toutes ces personnes ne sont pas des retraités, il est difficile de la qualifier de «migration de retraités ». La population migrante d'origine européenne, généralement mariée, détentrice de diplômes secondaires, a généralement autour de 50 ans et n'est donc pas «vieille» pour le contexte européen. Ces migrants, de plus en plus intéressés par l'achat de biens immobiliers, sont en train de s'installer de façon durable, en cherchant à se mettre en conformité avec les lois sur les étrangers, par l'obtention de permis de séjour touristiques renouvelables sur la durée ou de cartes de séjour. Parmi les facteurs attractifs, on relève les conditions climatiques, à l'instar d'autres migrations du même type vers d'autres pays (Warnes \& Patterson, 1998 ; Kaiser, 2001). Les migrants estiment que le climat méditerranéen d'Alanya est bien plus conforme à leurs aspirations que les climats froids et humides de leurs pays d'origine. Les conditions de vie, en particulier le 
coût de la vie, le caractère hospitalier et la chaleur des relations avec les autochtones, jouent un rôle secondaire non négligeable. Enfin, les opportunités d'emploi créées directement par cette migration, comme dans le secteur immobilier touristique à Alanya, ne doivent pas être négligées : ceci explique l'âge moyen finalement assez faible des résidents étrangers. Le maintien de la croissance du marché immobilier dans l'arrondissement tendrait à prouver que la migration va continuer dans les années à venir.

La migration des Européens a grandement affecté Alanya de diverses manières et a opéré une mutation. Le secteur immobilier connaît une revitalisation importante, les constructions neuves se multiplient et les impacts culturels commencent à se voir dans le paysage urbain. En raison d'une forte demande et de gains importants, le nombre d'agences immobilières a crû rapidement dans l'arrondissement. Les propriétaires d'agences sont généralement extérieurs à la région et n'en sont pas à leur première création d'entreprise. Parmi eux, les étrangers occupent une place non négligeable. En raison de la vitalité du secteur et de la forte demande de logements modernes, c'est toute la région côtière qui est affectée.

Les Européens ont importé leur mode de vie et leurs habitudes en Turquie et cherchent à maintenir et diffuser leurs cultures. En créant des espaces qui leur sont propres, en constituant diverses associations et un Conseil des résidents étrangers, véritables innovations dans le paysage turc, en transférant leur langue et leur religion (journaux locaux, espaces religieux), les migrants ont transformé la géographie culturelle locale et ont très vite imprimé leurs spécificités sur l'espace occupé.

Parallèlement à la création de relations avec l'Union européenne, la Turquie devra prouver aux yeux des Européens qu'elle est un pays stable et digne de confiance. Dans cette optique, la migration de personnes d'âge mûr mais encore professionnellement actives, ne sera pas sans importance. Elle est de plus en plus acceptée par l'opinion publique. Dans le cas de la candidature turque à l'UE, l'enjeu est ici majeur, car à l'image de ce que l'Espagne a connu lors de son adhésion, les prix de l'immobilier augmenteront rapidement et, à Alanya, les achats de biens immobiliers effectués par les Européens deviendront une forme d'investissement rentable. Dès lors, et face aux résultats produits par l'immigration que l'on a observés à Alanya, il y a une nécessité à envisager leurs effets sur le développement des côtes turques. Peut-on alors aussi imaginer en retour un renversement de l'image négative que garde la Turquie auprès de très nombreux Européens?

\section{BIBLIOGRAPHIE}

AKOVA İsmet (1999) Alanya'nın Turizm Olanakları [Les possibilités touristiques d'Alanya], Istanbul Üniversitesi Edebiyat Fakültesi Coğrafya Dergisi (7), pp. 183-207.

ALANYA (2003) Ekonomik Rapor [Alanya 2003 rapport économique], 2004, Alanya Ticaret ve Sanayi Odası, Antalya. 
ARMUTÇU Emel (2004) Alanya'daki Almanya [L'Allemagne à Alanya], National Geographic Türkiye, Juillet, pp. 38-48.

AYBAY Rona (2005) Yabancilar Hukuku [Droit des Etrangers], Istanbul Bilgi Üniversitesi

CASADO-DIAZ Maria Angeles (1999) Socio-demographic Impacts of Residential Tourism : A Case Study of Torrevieja, Spain, International Journal of Tourism Research (1), pp. 223-237.

Devlet Meteoroloji İşleri Genel Müdürlüğü (1974) Ortalama ve Ekstrem Kıymetler Meteoroloji Bülteni [Bulletin météorologique des valeurs moyennes et extrêmes], Başbakanlık Basımevi, Ankara.

KAISER Bianca (2003) Life Worlds of EU Immigrants in Turkey, in E. Zeybekoğlu and B. Johanson Eds, Migration and Labour in Europe: Views from Turkey and Sweden, MURCIR \& NIWL, Istanbul.

KAISER Bianca (2001) Internationale Ruhesitzmigration und wandelnde Ansprüche an das Lebensumfeld, Vortrag im Rahmen der Fachtagung Mobilität und gesellschaftliche Partizipation im Alter, 11.07.2001 and der TU Dresden.

KING Russel, WARNES M. A., WILLIAMS Allan M. (1998) International Retirement Migration in Europe, International Journal of Population Geography, (4) 2, pp. 91-111.

KING Russel, PATTERSON Guy (1998) Diverse Paths : The Elderly British in Tuscany, International Journal of Population Geography, (4) 2, pp. 157-182.

MUTLUER Mustafa (2004) Migrations vers la Turquie : survol des processus historiques et des données récentes, in A. Manço Ed., Turquie, vers de nouveaux horizons migratoires ?, L'Harmattan, Paris, pp. 85-107.

MÜLLER M. J. (1980) Handbuch ausgewähler Klimastationen der Erde, Universität Trier, Heft 5, Forschungsstelle Bodenerosion Mertesdorf (Ruwertal), Trier.

O’REILLY Karen (2000) The British and the Costa del Sol: Transnational Identities and Local Communities, Routledge, London.

ÖZGÜÇ Nazmiye (1977) Sayfiye Yerleşmeleri : Gelişme ve Başlıca Özellikler [Les lieux de villégiature : développement et principales caractéristiques] Istanbul Üniversitesi Coğrafya Enstitüsü Dergisi (22), pp. 143-162.

ÖZGÜÇ Nazmiye (2003) Turizm Coğrafyası : Özellikler ve Bölgeler [Géographie du Tourisme : Particularités et Régions], Çantay, Istanbul.

RODRIGUEZ Vicente, FERNANDEZ-MAYORALAS Gloria, ROJO Fermina (1998) European Retirees on the Costa del Sol : A Cross-national Comparison, International Journal of Population Geography, (4) 2, pp. 183-200.

SÜDAş İlkay (2005) Europe's New Destination for Cultural Contact : Alanya, Turkey, paper presented at VIII Congreso Cultura Europea, Centro de Estudios Eurpeos, Universidad de Navarra, Pamplona.

TURAN A. H., KARAKAYA E. (2005) Türkiye'ye Yabancı Emekli Göçü : Didim Örneği [La migration des retraités étrangers en Turquie : l'exemple de Didim], contribution à KEAS 05, II Kentsel Ekonomik Arastırmalar Semposyumu, Denizli.

WARNES M. A., PATTERSON G. (1998) British Retirees in Malta : Components of the CrossNational Relationship, International Journal of Population Geography, (4) 2, pp. 113-133.

WILLIAMS A., PATTERSON G. (1998) An Empire Lost but a Province Gained : A Cohort Analysis of British International Retirement in the Algarve, International Journal of Population Geography (4) 2, pp. 135-145. 
WILLIAMS A., HALL M. (2000) Tourism and Migration : New Relationship between Production and Consumption, Tourism Geographies, (2) 1, pp. 5-27.

YILMAZ Hakan (2005) Avrupa'lıların Türkiye'nin Gündelik Hayatı Hakkındaki Anlatıları : Türkiye'de Yaşayan Avrupa'lılarla Yapılan Görüşmeler [Ce que les Européens racontent sur leur vie quotidienne en Turquie : Entretiens avec les Européens vivant en Turquie], in H. Yılmaz Ed., Avrupa Haritasında Türkiye [La Turquie sur la carte de l'Europe], Boğaziçi Üniversitesi, Istanbul, pp. 20-39.

\section{Revue de presse et sites web}

Alanya Belediyesi [Municipalité d'Alanya], http://www.alanya-bld.gov.tr

Alanya Kaymakamlığı [Sous-préfecture d'Alanya], http://www.alanya.gov.tr

Anlaya'da Yerleşik Yabancılar, Sık Sık trafik Cezası yemekten Bıktı [Les étrangers installés à Alanya en ont assez de payer des contraventions], article paru le 20.07.2006 dans http:// www.haber.com (accès le 28.07.2006).

BAHAR H. İ. (2004) Türkiye'de Yerleşik Yabancılar : Alanyalı Almanlar [Les étrangers installés à Alanya : les Allemands d'Alanya], USAK- Uluslararası Stratejik Araştırmalar Kurumu.

HIELSCHER A. (2003) Hier bin ich wieder wer, Der Spiegel (42), 64-67.

Hürriyet, Yabancıların gelişiyle yaşam kalitemiz arttı [Notre qualité de vie à crû avec l'arrivée des étrangers], 21.01.2005.

Radikal, Alanya « tatlı vatan », 07.07.2003, www.radikal.com.tr

\section{NOTES}

1. Voir Olivier Dehoorne, Tourisme et migration, entre logiques des marchés et système des mobilités : revue bibliographique, (18) 1, accès par http://remi.revues.org/document2631.html.

2. NdT. Ce patrimoine est important et comprend en ville comme dans les environs des sites byzantins (églises Saint Georges et Hıdırellez), seldjoukides (forteresse, port et arsenal d'Alanya, forteresses et caravansérails) et plus rarement ottomans (mosquées et mausolées). Hormis plages, criques et grottes proches du littoral, le patrimoine naturel comprend des alpages et de belles forêts de conifères (cèdres) dans l'arrière-pays du Taurus.

3. Les retraités sont souvent très jeunes en Turquie où il faut cotiser 20 années pour bénéficier d'une retraite. Il n'est donc pas rare de rencontrer des retraités de moins de 50 ans. Mais l'espérance de vie reste très inférieure aux standards européens et la couverture sociale encore inférieure à ce qu'elle est en Europe occidentale (NdlR).

4. Contrairement aux Européens, les immigrés turcs indiquent assez facilement leurs niveaux de revenus (NdlR).

5. La Méditerranée turque, entre la péninsule de Datça et la ville de Mersin, se caractérise par de hautes montagnes calcaires plongeant dans la mer. La côte n'a été peuplée que récemment par les Turcs, alors qu'elle abrite des centaines de sites archéologiques antiques. La montagne, où l'on peut encore rencontrer des nomades yorük (artisanat du tapis) et tahtacı (nomades forestiers), est riche en sites naturels et historiques (NdlR).

6. Le statut de municipalité (belediye) n'est pas octroyé à toutes les agglomérations. Il faut dépasser 2000 habitants et fournir un certain nombre de services pour bénéficier de ce statut. Le belde est une extension urbaine non indépendante alors que mahalle (quartier d'une ville) et köy 
(village) ont le statut de muhtarlı (délégation) et élise un délégué faisant, entre autres tâches, l'inscription à l'État-Civil. Dans les régions urbanisées et touristiques, ces distinctions commencent à être caduques (NdlR).

7. Les villes turques sont subdivisées en quartiers (mahalle) disposant d'un représentant élu (muhtar) qui est en même temps officier d'État-Civil. Voir note 6 (NdlR).

8. À la demande d' ìlkay Südaş, Rainer Korten, habitant allemand d'Alanya répond qu'il serait insensé de vouloir établir une différence entre catholiques et protestants. Le prêtre est donc " œcuménique» et l'office commun aux deux Églises. Quant aux vieilles églises historiques d'Alanya, les rénover couterait fort cher et elles se trouvent mal situées pour les personnes les plus âgées, et donc peu accessibles. Hagios Georgios, qui garde des traces de fresques, est de construction byzantine et située à $10 \mathrm{~km}$ du centre. Elle n'a été abandonnée qu'en 1924, au départ des Karamanl, ces orthodoxes turcs écrivant le turc en caractères grecs, pris dans l'échange de populations du traité de Lausanne. Une autre église ancienne est visible dans les ruines de la forteresse seldjoukide. Aujourd'hui en ruine, elle aurait servi d'hôtel des monnaies (darphane). Elle fait partie du site classé de la citadelle d'Alanya.

9. Ces ouvertures de charcuteries sont évidemment dénoncées par les partis islamistes et nationalistes, mais à ce jour aucun incident sérieux n'est à déplorer. D'autres faits du même type ont été repérés par le traducteur à Marmaris, autre cité touristique de la côte méditerranéenne turque. Il existe des boucheries vendant de la viande de porc à Istanbul et Izmir depuis fort longtemps. Jusqu'à récemment, il $\mathrm{y}$ avait des dizaines d'élevages porcins en Thrace et près d'Izmit, mais en raison de la multiplication de tracasseries ces dernières années, seules quelquesuns ont réussi à se maintenir ( $\mathrm{NdlR})$.

10. NdT : Pour qui connaît la Turquie, ces petits problèmes prêtent à sourire... plus ou moins. Les fluctuations de prix selon la tête ou la nationalité du client sont classiques en Turquie (comme au Mexique pour les touristes américains). Chats et chiens ont rarement des maîtres, les Turcs y sont habitués et commencent à peine à laisser entrer des chiens dans leurs logements. Le bruit est une constante méditerranéenne connue (musique, animation nocturne, repas entre amis et voisins, conversations animées...). Quant aux plaques d'immatriculations, il s'agit des plaques bleues réservées aux non-ressortissants : les voitures ont une immatriculation turque, mais ne peuvent être vendues sur le marché turc. Il est souvent très difficile de les obtenir par la Préfecture, le fonctionnement du système, assez rigide, s'apparente à l'obtention de plaques vertes, diplomatiques. Nul doute que la réglementation devra changer rapidement dans les années à venir.

\section{AUTEURS}

\section{ILKAY SÜDAŞ}

Assistant de recherche ilkaysudas@yahoo.com, Département de Géographie de la Faculté des Lettres et Sciences Humaines de l'Université de l’Egée, Izmir-Bornova.

\section{MUSTAFA MUTLUER}

Professeur de Géographie mustafa.mutluer@ege.edu.tr, Département de Géographie de la Faculté des Lettres et Sciences Humaines de l'Université de l'Egée, Izmir-Bornova. 